\title{
Modeling methodology for stress determination by XRD in polycrystalline materials
}

S. Dufrenoy ${ }^{1, a^{*}}$, T. Chauveau ${ }^{1, b}$, R. Brenner ${ }^{2, c}$, C. Fontugne ${ }^{3, d}$, B. Bacroix ${ }^{1, e}$

${ }^{1}$ LSPM - CNRS, Université Paris 13, 99 av. J.B. Clément, 93430 Villetaneuse, France

${ }^{2}$ Institut Jean Le Rond d'Alembert, CNRS - Université Pierre et Marie Curie, UMR 7190, 4 place Jussieu, Paris, France.

${ }^{3}$ INEL, ZA Chemin départemental 405, 45410 Artenay, France.

${ }^{a}$ stephane.dufrenoy@lspm.cnrs.fr, ${ }^{b}$ thierry.chauveau@univ-paris13.fr, ${ }^{c}$ renald.brenner@upmc.fr, c.fontugne@inel.fr, ${ }^{\mathrm{e}}$ brigitte.bacroix@univ-paris13.fr

Keywords: self-consistent model, X-ray diffraction (XRD), texture, stresses.

\begin{abstract}
To get a quantitative estimate of residual stresses in polycrystals from XRD measurements, a micromechanical modeling is required, except in particular cases. The most widely used method is only valid for homogeneous and isotropic samples. We present here the possibility to determine residual stresses by coupling measurements with the portable INEL ${ }^{\mathrm{TM}}$ Xsolo equipment with a self-consistent polycrystalline model. This methodology may take into account texture and intergranular stresses induced by thermomechanical treatments. One example obtained for titanium subjected to tensile loading illustrates the methodology.
\end{abstract}

\section{Introduction}

Residual stresses are important for structural materials, since they are generally present after all thermomechanical treatments and may have beneficial or detrimental effects on the overall behavior and life time of the materials. They are usually determined by X-ray diffraction. However, the classical procedure usually implies a large number of individual measurements as well as very restricting hypotheses [1]. In the present paper, we present a recently developed measurement method that we illustrate by a study case obtained on a titanium alloy, and validate through a comparison with calculations obtained by polycrystalline models.

\section{Brief summary of usual definition of residual stresses}

Polycrystalline samples usually contain residual stresses after a thermomechanical treatment, whose origin may be plasticity, thermal dilatation or phase transformation; due to the polycrystalline nature of the materials, these stresses vary from point to point within the microstructure [2]. Thus, several orders of stresses are generally distinguished [2,3]. The first order stress is defined as the average stress on a given volume containing a large number of grains (and which has to be distinguished from the sample, see Fig.1).

$$
\boldsymbol{\sigma}_{V}^{I}=\frac{1}{V} \cdot \int_{V} \boldsymbol{\sigma}(x) d V \text { with } \boldsymbol{\sigma}(x) \text { being the local stress at point } x \text { of the sample. }
$$

The average of this stress on the whole sample is equal to 0 , when it is unloaded. This stress is also equal to 0 if the sample is homogeneous. We can also define an average stress per grain.

$$
\boldsymbol{\sigma}_{g}=\frac{1}{V_{g}} \cdot \int_{V_{g}} \boldsymbol{\sigma}(x) d V
$$

The $2^{\text {nd }}$ order stress is then defined as the difference between the grain stress and the $1^{\text {st }}$ order stress

$$
\boldsymbol{\sigma}_{g}^{I I}=\boldsymbol{\sigma}_{g}-\boldsymbol{\sigma}_{V}^{I}
$$


Again, the average of this stress on the volume $V$ of the investigated part of the material is equal to 0 . We can then also define a third order stress $\sigma_{s g}^{I I I}$, which results from the heterogeneity within single grains, at the level of a subgrain or dislocation cell, but this will be neglected here.

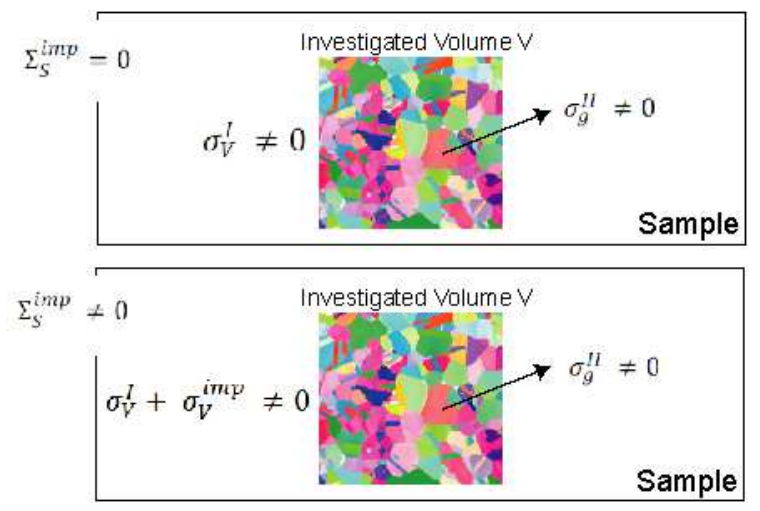

Fig.1. Illustration of the first and second order residual stresses for (a) an unloaded or (b) a loaded sample.

Please note that the value of the stress imposed on the investigated volume may differ from the macroscopic one, when the sample is heterogeneous.

These residual stresses are associated with residual elastic strains within the material. In the case of an unloaded sample, we can consider that the investigated volume, which presents an elastoplastic behavior, is thus subjected to a stress $\boldsymbol{\sigma}_{V}^{I}$ in the elastic range. And we can write:

$$
\boldsymbol{\sigma}_{g}=\boldsymbol{B}_{g}: \boldsymbol{\sigma}_{V}^{I}+\boldsymbol{\sigma}_{g}^{I I}
$$

The average local (residual) stress at the grain level thus comprises the contribution of both first (elastic incompatibilities) and second order (created by the prior plastic strain) residual stresses. In this expression, the tensor $\boldsymbol{B}_{g}$ is the so-called localization tensor, which depends on both the microstructure of the material and its elastic behavior. It can be calculated by a homogeneization model (such as Voigt, Reuss or Self - Consistent [4]), as long as the local elastic constitutive law is known. If we neglect the intragranular heterogeneities, this law can be written as:

$$
\boldsymbol{\varepsilon}_{g}=\boldsymbol{S}_{g}: \boldsymbol{\sigma}_{g}
$$

with $\boldsymbol{S}_{g}$ the so-called compliance tensor. From this we get the expression of the grain elastic strain

$$
\varepsilon_{g}=S_{g}: B_{g}: \sigma_{V}^{I}+S_{g}: \sigma_{g}^{I I}
$$

If we average now on one diffraction volume $\Omega$, associated with a diffracting plane $\{\mathrm{hkl}\}$ and a diffraction vector (unit vector $\mathbf{K}$ ) and project on the diffraction vector, we get:

$$
\left\langle\varepsilon_{K K}\right\rangle_{\Omega}=\mathbf{K} \times \mathbf{K}:\left\langle\boldsymbol{S}_{g}: \boldsymbol{B}_{g}\right\rangle_{\Omega}: \boldsymbol{\sigma}_{V}^{I}+\mathbf{K} \times \mathbf{K}:\left\langle\boldsymbol{S}_{g}: \boldsymbol{\sigma}_{g}^{I I}\right\rangle_{\Omega}
$$

This is what is usually measured by X-ray diffraction (the components of the vector $\mathrm{K}$ are usually expressed as a function of the two angles $\varphi$ and $\psi$ with respect to the sample reference system). The first term is linked to the elastic localization of the macroscopic applied stress, and the second term depends on the distribution of residual stresses in the material. The general expression of both terms is usually not simple, but can be estimated by a homogenization method, as long as we know the origin of the residual stresses (plasticity, thermal dilatation or phase transformation) and are able to calculate their distribution after a given thermomechanical treatment.

In terms of measurements by X-ray, the classical methods, whose aim is to determine the sole macrostress tensor $\boldsymbol{\sigma}_{V}^{I}$, always consider that the second term can be neglected (which is indeed difficult to check); Eq. 8 can then be rewritten as:

$$
\left\langle\varepsilon_{K K}\right\rangle_{\Omega}=\boldsymbol{F}(\mathbf{K}): \boldsymbol{\sigma}_{V}^{I}
$$


They also assume that, in the case of a biaxial stress tensor, $\left\langle\varepsilon_{K K}\right\rangle_{\Omega}$ is a linear expression with respect to $(\sin \psi)^{2}$, which allows a simple determination of the macrostress tensor. Indeed this last assumption is valid in only two restricting cases:

1. The material is elastically isotropic; in this case, it is easy to show that the term $\boldsymbol{F}(\mathbf{K})$ does not depend on the diffracting plane and volume, but depends solely on the macroscopic elastic constants which are known a priori. Thus, only one peak and one $\varphi$ value are needed.

2. The material is elastically anisotropic at the level of the single crystal, but macroscopically isotropic (no texture). The macroscopic elastic constants are replaced by the so-called Diffraction Elastic Constants (DEC) which depend on the diffracting plane and the texture of the material [3]. In this case, they have to be estimated by a calculation or evaluated from several measurements.

We can also measure elastic strains during a tensile test. If the specimen is loaded in the elastic range, and if we assume that the investigated volume is also elastically loaded, Eq. 8 becomes:

$$
\left\langle\varepsilon_{K K}\right\rangle_{\Omega}=\mathbf{K} \times \mathbf{K}:\left\langle\boldsymbol{S}_{g}: \boldsymbol{B}_{g}\right\rangle_{\Omega}: \boldsymbol{\sigma}_{V}^{I}+\mathbf{K} \times \mathbf{K}:\left\langle\boldsymbol{S}_{g}: \boldsymbol{\sigma}_{g}^{I I}\right\rangle_{\Omega}+\mathbf{K} \times \mathbf{K}:\left\langle\boldsymbol{S}_{g}: \boldsymbol{B}_{g}\right\rangle_{\Omega}: \boldsymbol{\sigma}_{V}^{i m p}
$$

which gives

$$
\left\langle\varepsilon_{K K}\right\rangle_{\Omega}(\text { loaded })=\left\langle\varepsilon_{K K}\right\rangle_{\Omega}(\text { unloaded })+\boldsymbol{F}(\mathbf{K}): \boldsymbol{\sigma}_{V}^{\text {imp }}
$$

Please note that in the case of an heterogeneous sample, the value of the stress $\boldsymbol{\sigma}_{V}^{i m p}$ imposed on the volume $V$ differs from the macroscopic one imposed on the sample $\Sigma_{S}^{i m p}$.

For a given model (which considers only the elastic behavior of the material), Eq. (11) allows us to compare easily calculated and experimental values of stresses, without being obliged to simulate the whole thermomechanical treatment at the origin of the residual stresses. This comparison enables then either to validate the experimental procedure (if the model can be considered as sufficiently predictive) or the model (if the experimental procedure is sufficiently precise). The aim of the present work is first to validate a new experimental procedure.

\section{Experimental material and methodology}

The investigated material is a Ti Alloy containing 3\% $\mathrm{Al}$ and 5\% V (in weight \%). This asreceived material in the shape of cold rolled and annealed sheets of $1 \mathrm{~mm}$ thickness contains both hexagonal and cubic phases. However, the content of the cubic phase is low (i.e. less than 10\%) and will be neglected. This Ti alloy has been received in the shape of cold rolled and annealed sheets of $1 \mathrm{~mm}$ thickness. The initial texture has been determined by XRD (Fig. 2). The obtained texture, moderately developed, is typical of cross-rolling in these materials.

Then, for stresses measurements, X-ray diffraction data were collected using a recently developed INEL ${ }^{\mathrm{TM}}$ Xsolo portable diffractometer in Bragg-Brentano geometry. The main parts of the experimental setup are: a point focus collimated $\mathrm{Cu}$ source $(4 \mathrm{~W})$ and a $2 \mathrm{D}$ CCD detector $(24 \times 34$ $\mathrm{mm}^{2}, 20 \mu \mathrm{m}$ step size, $2 \theta$ range: $106^{\circ}-173^{\circ}$ ) equipped with a $\mathrm{Ni}$ filter in order to suppress the $\mathrm{k}_{\beta}$ radiation. This device is placed on a $\varphi$ rotation axis. Before any measurement, a calibration is done with a LaB6 (NIST SRM 660A) powder. With a fixed $\omega=55^{\circ}$ incident angle for the X-ray beam source, samples are placed in a tensile test device (developed in LSPM) at a distance of $30 \mathrm{~mm}$ from both the source and the center of the detector.
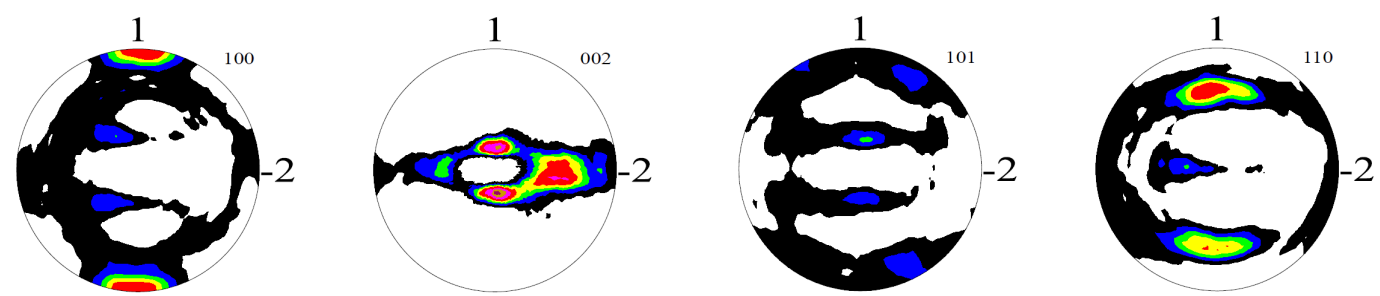

Fig. $2\{10 \overline{1} 0\},\{0002\},\{10 \overline{1} 1\}$ and $\{11 \overline{2} 0\}$ recalculated pole figures using the LABOTEX software [5]; the maximum value equal to 6.32 is obtained on the $\{0002\}$ pole figure. 
Measurements are performed on unloaded or loaded samples (Fig. 3). The tests are performed at various directions within the rolling plane and X-ray measurements are performed during the tests at various stress values, both in the elastic range and in the plastic range (only the elastic part will be used below). The obtained curves are presented in Fig. 4; it is seen that the anisotropy in the elastic range is not very strong and the material seems to be in a hardened state, which means that recrystallization has not been complete during annealing and that the material contains most probably second order stresses of plastic origin.

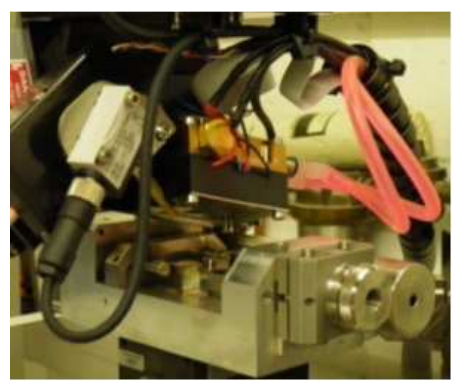

Fig. 3. Experimental set-up: Xsolo and tensile machine.

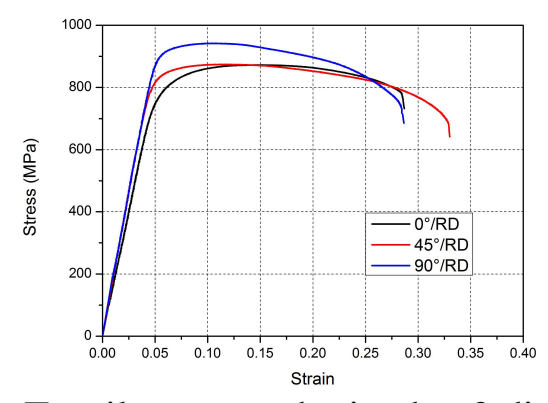

Fig. 4. Tensile curves obtained at 3 directions with respect to the rolling direction $(\mathrm{RD})$.

We will analyse below the data obtained at $600 \mathrm{MPa}$ (i.e. in the elastic range) for the RD tensile test. The 2D measurements and subsequent analysis are performed for both unloaded and loaded samples in $2 \varphi$ sample positions, namely 0 and $90^{\circ}$ (Fig. 5). The obtained measurements correspond to several peaks and several values of the angles $\varphi$ and $\psi$ simultaneously (more precisely $\varphi$ and $\eta$ experimental angles which are easily converted into commonly used $\varphi$ and $\psi$ ones).
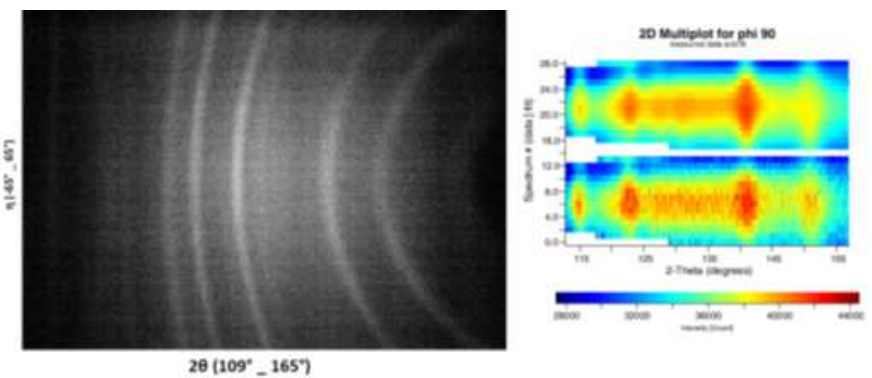

Fig. 5. (a) 2D experimental data. (b) MAUD Rietveld analysis.

The extraction of the elastic strains $\left\langle\varepsilon_{K K}\right\rangle_{\Omega}$ from the experimental data is performed through a Rietveld analysis of the whole set of data using the MAUD software [6], and neglecting the second order stresses. The measured experimental texture of the material can be taken into account in the MAUD analysis in order to calculate the DEC with simple models as Voigt and Reuss.

In the analysis of an unloaded sample, the initial lattice components as well as the various stress components of the $\boldsymbol{\sigma}_{V}^{I}$ tensor can be left free. The output will thus give us an estimate of the initial stress state of the material and a precise evaluation of the lattice parameters. For the studied material, considering $\mathrm{C}_{11}=\mathrm{C}_{22}=168, \mathrm{C}_{33}=190.5, \mathrm{C}_{44}=\mathrm{C}_{55}=48.8, \mathrm{C}_{66}=36.75, \mathrm{C}_{13}=\mathrm{C}_{23}=69.3$, and $\mathrm{C}_{12}=94.5$ for the single crystal elastic constants $(\mathrm{GPa})$, the analysis is first performed without imposing the lattice parameters using the two Voigt and Reuss models for the calculation of the DEC, then these parameters are set equal to the average values of the two sets found previously and only the stress components are adjusted by considering a biaxial stress. The output of this analysis is given in Table 1.

Table 1. Lattice parameters and stress components obtained for the unloaded sample.

\begin{tabular}{|c|c|c|}
\hline DEC calculation & Fixed lattice parameters $(\AA)$ & $\boldsymbol{\sigma}_{11}^{I}, \boldsymbol{\sigma}_{22}^{I}, \boldsymbol{\sigma}_{12}^{I}(\mathrm{MPa})$ \\
\hline Voigt model & \multirow{2}{*}{$\bar{a}=2.93605 \pm 2 \mathrm{E}-4, \bar{c}=4.680 \pm 9 \mathrm{E}-4$} & $-367 \pm 27,-212 \pm 25,-106 \pm 11$ \\
\hline Reuss model & & $-227 \pm 19,-238 \pm 24,-63 \pm 9$ \\
\hline
\end{tabular}


The initial stress state is thus far from 0 , which confirms the fact that the material was not in a recrystallized state. Some $2^{\text {nd }}$ order stresses are thus also present in the material, but are not estimated here. This necessarily leads to a first source of error on the estimation of the $1^{\text {st }}$ order stresses. The output of the analysis performed for the loaded sample (with a macroscopic imposed stress equal to $600 \mathrm{MPa}$ ) is presented in Table 2 .

Table 2. Lattice parameters and stress components obtained for the loaded sample.

\begin{tabular}{|c|c|c|}
\hline DEC calculation & Fixed lattice parameters $(\AA)$ & $\boldsymbol{\sigma}_{11}^{\prime I}, \boldsymbol{\sigma}_{22}^{\prime I}, \boldsymbol{\sigma}_{12}^{\prime I}(\mathrm{MPa})$ \\
\hline Voigt model & \multirow{2}{*}{$\bar{a}=2.93605 \pm 2 \mathrm{E}-4, \bar{c}=4.680 \pm 9 \mathrm{E}-4$} & $766 \pm 22,-22 \pm 21,135 \pm 9$ \\
\hline Reuss model & & $601 \pm 17,-311 \pm 23,96 \pm 7$ \\
\hline
\end{tabular}

For the loaded sample, the measured stresses are the superposition of the residual stresses present within the material before loading (Table 1) and of the stress applied to the investigated volume. This last one may differ from the one applied to the whole sample $(600 \mathrm{MPa})$, since the material is heterogeneous. The estimation of this imposed stress, using the two extreme models for the calculation of the DEC, gives e.g. a range of 828 to $1133 \mathrm{MPa}$ for the component $\boldsymbol{\sigma}_{11}^{\mathrm{imp}}$.

\section{Calculations using a Self-Consistent Model}

The elastic SC model, initially developed by Kröner [4], and further extended to the treatment of thermo-elasto-plasticity by Hutchinson [7] and Turner et al. [8] has been used here, since it has been shown to be well adapted to the calculation of $1^{\text {st }}$ order elastic stresses. It will be used for three purposes: (i) to investigate to which extent Eq. (10) can be approximated by a linear relationship as a function of $(\sin \psi)^{2}$ for elastically loaded anisotropic materials (initially stress-free), (ii) when it is the case, how good is the estimation of the stress from obtained linear curves and (iii) to compare experimental and calculated lattices strain in the case of an elastically loaded sample using Eq. (11).

In order to do so, an aggregate of 186624 orientations either randomly distributed within the Euler space (isotropic texture) or representative of the measured texture of the investigated Ti alloy (anisotropic texture) is subjected to a tensile test up to 600MPa. When this value is reached, the resulting elastic strain is calculated with the SC model for several families of diffraction planes and several values of the two angles $\varphi$ and $\psi$. One example of such calculations is presented in Fig. 6 for the $\{21 \overline{3} 3\}$ reflection peak, which is the one recommended by the current standard for hexagonal materials [1]. Note that the calculated strain $\left\langle\varepsilon_{K K}\right\rangle_{\Omega}$ is simply equal here to $\boldsymbol{F}(\mathbf{K}): \boldsymbol{\sigma}_{V}^{i m p}$, with $\boldsymbol{\sigma}_{V}^{i m p}=600 \mathrm{MPa}$. It is clear that when the material is macroscopically isotropic, both curves are linear, but one of them becomes slightly non-linear for the anisotropic material. The various slopes reflect also the deviation from isotropy. Now, if we want to apply the standard stress determination to these curves, we can calculate using the model the DEC from the texture and from a priori selected elastic constants for the single crystal and then from the linear fit of the curves extract the value of stress. The results of this analysis are presented in Table 3.
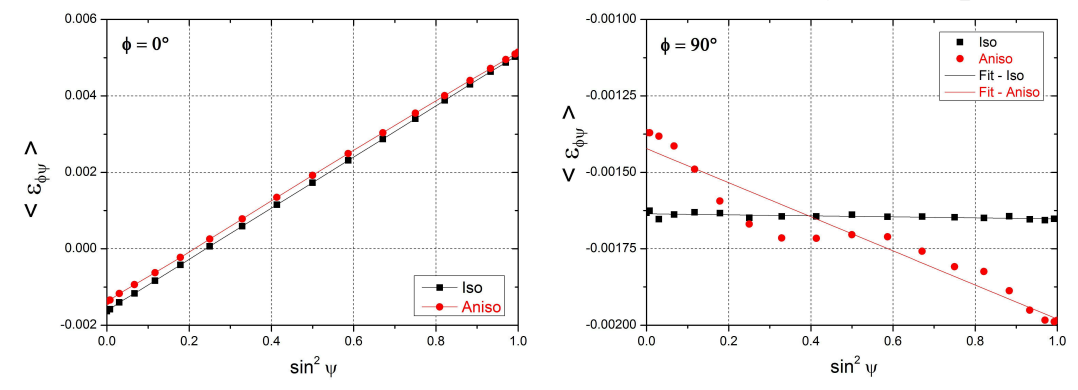

Fig. $6\left\langle\varepsilon_{\varphi \psi}\right\rangle$ as a function of $(\sin \psi)^{2}$ for 2 values of $\varphi$ for the $\{21 \overline{3} 3\}$ reflection calculated with the SC model, for 2 textures (anisotropic or isotropic) with anisotropic elastic constants. The slope of these curves mainly reflect the value of $\boldsymbol{\sigma}_{11}^{\text {imp }}$ for $\varphi=0$ and of $\boldsymbol{\sigma}_{22}^{i m p}$ for $\varphi=90$.

There is a deviation from the expected value of $600 \mathrm{MPa}$ in most of the cases. A better agreement is hopefully found when the assumptions made on the EC do correspond to the one used in the SC calculation. It is also worth noting that the error made is less for the recommended peak than for any other one, like e.g. the $\{0006\}$ one, where the error can exceed $20 \%$. 
Table 3. Recalculated stress values $\boldsymbol{\sigma}_{11}^{i m p}$ (in MPa) from the linear fit of the SC response (Fig. 6) for various hypotheses concerning the texture and elastic constants

\begin{tabular}{|c|c|c|c|c|c|}
\hline & & \multicolumn{2}{|c|}{$21 \overline{3} 3$} peak & \multicolumn{2}{c|}{0006} peak \\
\hline Texture & Elastic Constants & $\varphi=0$ & $\varphi=90$ & $\varphi=0$ & $\varphi=90$ \\
\hline \multirow{2}{*}{ Isotropic } & Isotropic & 610 & 588 & 518 & 485 \\
\cline { 2 - 6 } & Anisotropic & 599 & 600 & 600 & 601 \\
\hline \multirow{2}{*}{ Anisotropic } & Isotropic & 596 & 618 & 512 & 468 \\
\cline { 2 - 6 } & Anisotropic & 607 & 597 & 614 & 603 \\
\hline
\end{tabular}

Now, we can compare the values of the elastic strains predicted by the SC model to the ones obtained by MAUD using Eq. (11). In this case, calculations are performed by imposing a macroscopic stress either equal to $600 \mathrm{MPa}$ like previously or to $980 \mathrm{MPa}$ (average value of the two found by MAUD for two different hypotheses for the calculation of the DEC). The result of this analysis is presented in Fig. 7.

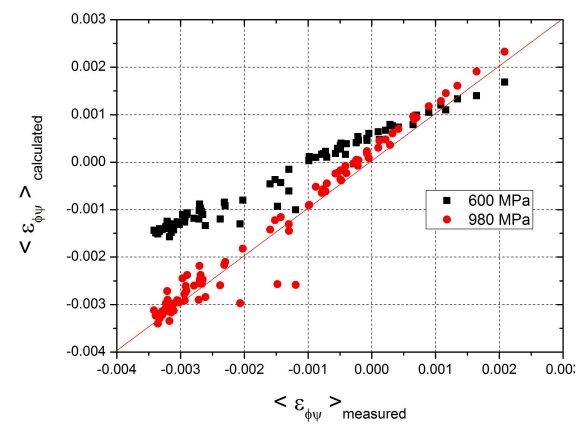

Fig. 7. Comparison of calculated and experimental elastic strains measured during a tensile test (macroscopic imposed stress $=600$ $\mathrm{MPa})$.

The agreement between experimental and calculated values is much better when the imposed stress value does correspond to the one which has been experimentally measured on the investigated volume rather than the one imposed on the whole sample. There is some remaining discrepancy for the negative strain values. These cases are in fact associated with much smaller diffracting values than the other measurements; for this reason, some of these measurements should be taken out from the analysis for this particular texture.

\section{Conclusions}

The present paper has reported the first residual stress measurements performed with the INEL Xsolo device on a titanium alloy under stress. It has been shown that this equipment allows reliable strain measurements using a multiple peak analysis to extract the strain and stress values. We have also illustrated the shortcomings of the standard stress measurement method and proven the capacity of the SC model to precisely evaluated strains and stresses for anisotropic materials.

Acknowledgments. The authors would like to thank the CG93 for the attribution of a $\mathrm{PhD}$ fellowship as well as the financial support of the IFR Paris Nord Plaine de France. ANR and CGI are gratefully acknowledged for their financial support of this work through Labex SEAM.

\section{References}

[1] AFNOR norm NF EN 15305, « Non-destructive Testing — Test Method for Residual Stress analysis by X-ray Diffraction ».

[2] Th. Bretheau and O. Castelnau in Proc. National Conference Rx\&Matière 2006, 121-152.

[3] A. Baczmanski, N. Hfaiedh, M. François, K. Wierzbanowski, Mater. Sci. Eng. A501 (2009), $153-165$.

[4] E. Kröner, Z. Phys. 151 (1958), 504.

[5] Labotex - Texture analysis software, http://www.labosoft.com

[6] Maud - Materials Analysis Using Diffraction, http://www.ing.unitn.it/ maud/.

[7] J.W. Hutchinson, Proc. R. Soc. Lond. A 319 (1970), 247.

[8] P.A. Turner, N. Christodoulou, C.N. Tomé, Int. J. Plasticity, 11 (1995), 251 - 265. 